\title{
IMAGE DETECTIVE: EFFICIENT IMAGE RETRIEVAL SYSTEM
}

\author{
N. D. Pawar', V. H. Sakore ${ }^{2}$, P. N. Shendage ${ }^{3}$, N. K. Shevate ${ }^{4}$ \\ ${ }^{1}$ Student, Computer Department, Bharati Vidyapeeth's College of Engineering for Women, Maharashtra, India \\ ${ }^{2}$ Student, Computer Department, Bharati Vidyapeeth's College of Engineering for Women, Maharashtra, India \\ ${ }^{3}$ Student, Computer Department, Bharati Vidyapeeth's College of Engineering for Women, Maharashtra, India \\ ${ }^{4}$ Student, Computer Department, Bharati Vidyapeeth's College of Engineering for Women, Maharashtra, India
}

\begin{abstract}
With the wide - spread use of image retrieval in various areas such as crime investigation, medical diagnosis, intellectual property rights, etc, today's need is to enhance the image retrieval process. In our research, we are combining Text Based Image Retrieval (TBIR) method with Content Based Image Retrieval (CBIR) method to enhance image retrieval. The base of CBIR is to extract different image features, such as Color, Shape and Texture. To improve the accuracy, we are using combination of most efficient feature extraction algorithms. We are using RGB to Lab conversion for color feature extraction, Modified Canny edge detection algorithm with variable sigma for shape feature extraction, Framelet transform method for texture feature extraction. For improving the speed of image retrieval process using TBIR, we are implementing automatic annotation technique. Images are annotated automatically without human intervention. It improves speed. Approximately one to two thousand images are stored in the database. Features are extracted from these images and stored into the database. Query images are processed in the similar way and similarity matching between query and database images is done through Hybrid Graph method. For that purpose, we have to generate image to image graph from extracted feature vectors and image to tag graph from database. Combining both these graphs, we get the Hybrid graph. Thus, the process of image retrieval is becoming efficient in both terms accuracy and time. Also, user can give input in terms of query image or textual query or sketch. This improves human-friendliness of this system.
\end{abstract}

Keywords: feature extraction, Lab, Modified Canny detection, Framelet transform, automatic annotation, similarity matching, Hybrid Graph, etc.

\section{INTRODUCTION}

Our paper is divided into three sections. First section gives introduction about image retrieval process. Second section describes literature review about various feature extraction methods and third section gives a brief description about our proposed system.

With the increase in size of digital data, it is very important to get appropriate data at particular time. Similarly image retrieval process is also important to get a particular image from very large database. Two main methods of image retrieval are: TBIR and CBIR. The main idea behind CBIR (Content Based Image Retrieval) is to extract features of images like color, texture and shape [1].Use of these hybrid features color, texture and shape as feature vectors gives better results [2]. But, now-a-days, with the increasing scope and applications of this field, it is important to enhance efficiency of image retrieval. Hence, our aim is to build a system which effectively retrieves similar images from a very large database by using TBIR and CBIR together.

Image retrieval process is basically three step process. First step is preprocessing on images. Then feature extraction and last step is similarity matching. In next section, we have described research work on this process.

\section{LITERATURE REVIEW}

In color images, color plays very vital role. There are various color spaces - RGB, HSV, HSL, CMYK, CIE Lab, CIE XYZ. According to Madhura $\mathrm{C}$ and Dheeraj D, converting RGB images to any other color spaces such as Gray, HSV, HIS, Lab, YCbCr, CMY and then processing these images gives good results [3]. Lab color model contains L i.e. Lightness factor, which matches human perception of lightness and provides very good results.

We know that, edge detection is very important as good edge detection improves other processing also. There exist many edge detection algorithms like Marr-Hilderth edge detector, Local Threshold, Boolean Function based edge detector and canny edge detector. Raman Maini and Dr. Himanshu Aggarwal studied different edge detection algorithms and finally they reached to the conclusion that Canny's edge detection algorithm performs better than all other edge detecting operators under almost all scenarios [4]. Even though it is more costly than others, it gives best results [5]. Hence, various modifications are done to improve efficiency of canny edge detection algorithm. Biman Debbarma and Dibyendu Ghoshal also proposed one modification. In their modified canny edge detection algorithm, variable sigma values are applied to different parts of image [6]. 
Texture gives visual characteristic of surface. Various feature extraction methods are Grey Level Co-occurrence Matrices, Local Binary Pattern, Gabor filters. S.Sulochana and R.Vidhya combined two methods of texture feature extraction: Framelet transform and Gray level co-occurrence matrix (GLCM). They compared the results of these methods for image retrieval [7].

Automatic Annotation is effective way for CBIR. Manual annotation is very time consuming and costly method. Though automatic annotation is less reliable than manual, speed is the advantageous factor [8].

Various distance measure techniques are available for similarity matching purpose, such as Euclidean distance, Canberra distance, Minkowski distance.

\section{PROPOSED SYSTEM}

We have seen different algorithms for various feature extractions. From our literature review, we get few of the most efficient feature extraction algorithms of corresponding features. To accomplish our goal of enhancing efficiency of image retrieval, we are combining these all algorithms in our proposed system. Hence we are using following algorithms:

a. RGB to Lab conversion: Color feature.

b. Modified canny edge detection algorithm with variable sigma: Shape feature.

c. Framelet Transform: Texture feature.

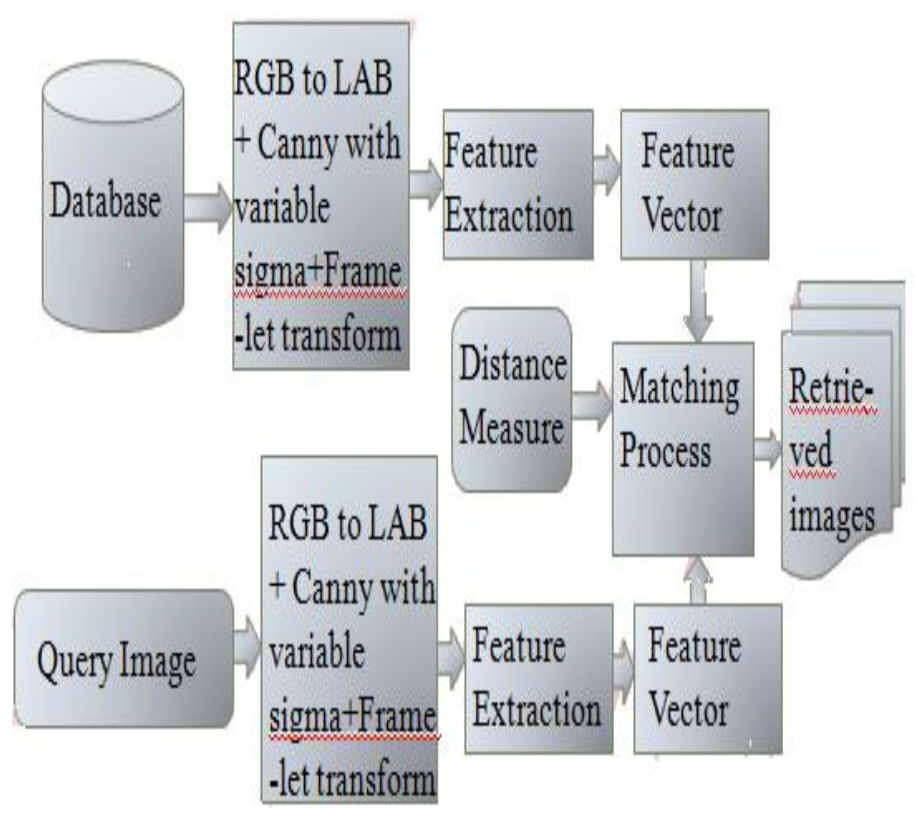

Fig -1: System Architecture

Fig - 1 represents the system architecture.

In some TBIR systems, annotations are given manually. There are disadvantages of this:

- More human resources are required to give manual annotation.
- According to human perception, there may be inconsistency in annotations [9].

Hence, we are providing automatic annotation to get rid of this problem.

We can provide input as sketch or image or textual query. For that purpose, we have to add following functionalities in our system:

- $\quad$ To retrieve images by using image as input, we have to use combination of most efficient feature extraction algorithms.

- To retrieve images by using sketch, the informational gap between sketch and colored image database must be handled [10].

- $\quad$ To retrieve images by using textual query, TBIR methods also must be incorporated.

\subsection{Feature Extraction}

\subsubsection{Color Feature Extraction}

As we know, converting RGB images to Lab gives better results. We are converting RGB to Lab.

Fig - 2 represents Lab color model.

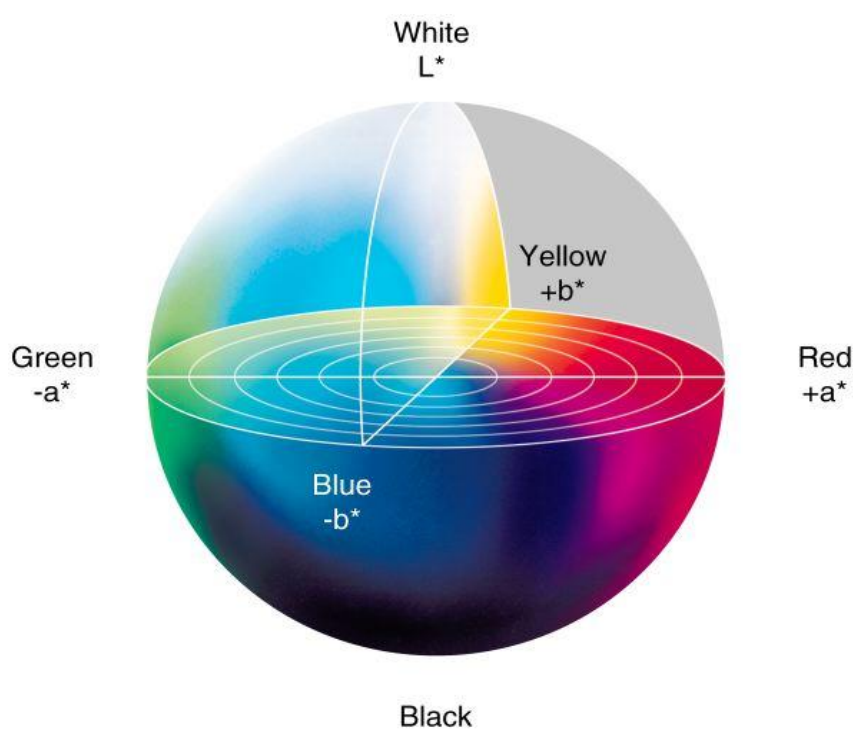

Fig -2: Lab color model

As, in the fig., $\mathrm{L}^{*}: 0$ - Black and 100 - diffuse white. $\mathrm{a}^{*}$ : negative values- green and positive values- magenta. $\mathrm{b}^{*}$ : negative values- blue and positive values- yellow.

Lab color space is a color space with dimensions, L for lightness, $\mathrm{a}$ and $\mathrm{b}$ are color opponent dimensions. Lab color space is computed using XYZ color space, but it is perceptually more uniform. Perceptually uniform means a change of the same amount in color value should produce a change of same visual importance [11]. This is one advantage of Lab color model and second advantage is that it is device independent. 
We cannot convert RGB to Lab directly. We have to convert it to XYZ first. Because, RGB is device dependent and Lab is device independent.

Algorithm:
i. Obtain $\mathrm{R}, \mathrm{G}$ and $\mathrm{B}$ values from the image.
ii. From these values obtain $\mathrm{X}, \mathrm{Y}$ and $\mathrm{Z}$ values.
iii. Then obtain $L, a$ and $b$ values by applying formulae to $\mathrm{X}, \mathrm{Y}$ and $\mathrm{Z}$ values.

\subsubsection{Shape Feature Extraction}

Canny algorithm is very much effective because of its features like good detection i.e. minimum false edges and important edges should not be missed, good localization i.e. closeness of real and detected edge, one response i.e. one edge produces once only.

Canny algorithm is a step-by-step process:

i. Image smoothing by Gaussian filter.

ii. Gradient calculation using Sobel operator.

iii. Non-maxima suppression.

iv. Thresholding with hysteresis.

v. Final binary image.

The Gaussian function is given by the formula,

$$
\mathrm{h}(\mathrm{x}, \mathrm{y})=\exp \left(-\pi\left(\mathrm{x}^{2}+\mathrm{y}^{2}\right) / \sigma\right)
$$

where the parameter $\sigma$ (sigma) indicates the width of filter that means degree of blurring. We can say that value of sigma is directly proportional to degree of blurring. But this approach gives average results in cases where prominent edges and faint edges both are of great significance. If the value of sigma is very high then faint edges will not be detected and if value of sigma is very low then noise may also get detected as edges. Hence, for every part of the image we are using different sigma values.

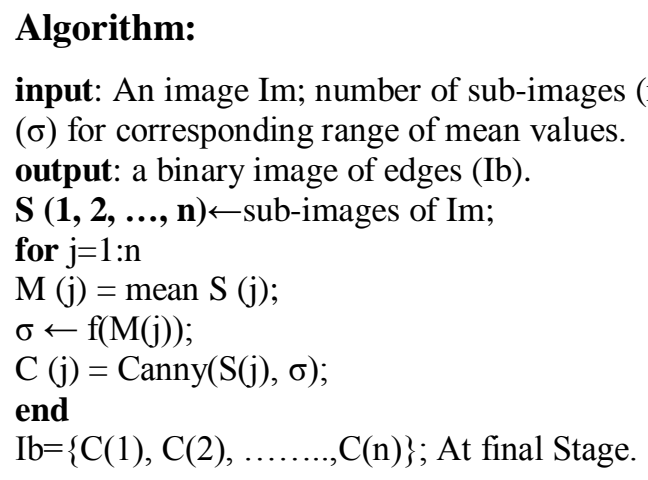

\subsubsection{Texture Feature Extraction}

Texture refers to properties that represent the surface or structure of an object. Two main texture description approaches exist: statistical and syntactic. With texture analysis, we can classify objects based on the underlying texture characteristic. Texture features statistics are classified into first-order, second-order and higher-order statistics. We are using statistical approach - Framelet transform.
The Proposed algorithm using Framelet Transform:

a. Decompose each image in Framelet Transform Domain.

b. Calculate the energy, mean and standard deviation of the Framelet transform decomposed image using following formulae.

$$
\begin{aligned}
& \text { Energy }=1 / M \times N \sum^{{ }^{\mathrm{M}=1}} \sum^{\mathrm{N}}{ }_{\mathrm{j}=1}\left|W_{k}(i, j)\right| \\
& \text { Standard Deviation }(\boldsymbol{\sigma} \boldsymbol{k})=\sqrt{ } 1 /(M \times N) \quad \sum_{\mathrm{i}=1}^{\mathrm{M} \sum_{\mathrm{j}=1}^{\mathrm{N}}\left(W_{k}\right.} \\
& \quad(i, j)-\mu k)^{\wedge} 2
\end{aligned}
$$

$\mu_{k}$ is Mean value of the $k t h$ Framelet transform sub band. $W_{k}$ is Coefficient of $k t$ h Framelet transform sub band. $M \times N$ is the size of the decomposed sub band.

c. The resulting $f=[\sigma 1, \sigma 2, \ldots . \sigma n, E 1, E 2 \ldots \ldots E n]$ is used to create the feature database.

d. Apply the query image and retrieve the feature vector as given in step b \& c.

e. Calculate the similarity measure using distance measuring scheme.

f. Retrieve all relevant images to query image based on minimum distance.

\subsection{Similarity Matching}

\section{Hybrid Graph:}

Build the image similarity graph by using the cosine function:

$$
\operatorname{Sim}(\mathrm{dp}, \mathrm{dq})=\frac{\mathrm{dp} \cdot \mathrm{dq}}{\|\mathrm{dp}\| \cdot\|\mathrm{dq}\|}
$$

Where $\mathrm{dp}$ and $\mathrm{dq}$ represent image feature vector of corresponding images dp and dq [12].

On the basis of the features extracted from the images, we match the similarity between the images by using hybrid graph. For that purpose, we build the image to image graph from extracted features \& image to tag graph from the database and by combining both graphs we will generate the bipartite graph to match the similarity.

\subsection{Automatic Annotation}

The image automatic annotation consists of many techniques that aim to find correlation between low level visual features and high level semantics. The main challenge is to create a model able to assign visual terms to an image to successfully describe it.

There are three methods for annotation: Manual, semi automatic and automatic. But automatic annotation saves time.

The first step in this algorithm is to create a training set of images that have been already annotated by humans. The next step is to extract same features from query image in 
order to compare it with all previously created models. The result yields probability value of each keyword present in the query image [13].

\section{CONCLUSION}

The proposed image retrieval system is very effective in terms of both accuracy and time. By using various efficient algorithms for feature extraction, we have achieved accuracy. We are eliminating the drawback of TBIR by using automatic image annotation. By using Text Based and Content Based image retrieval processes together, we are giving user the choice to get similar images by using image or text or sketch. Finally by using graphs obtained from CBIR and TBIR we generate a hybrid graph, which gives better results concerning accuracy and time.

\section{ACKNOWLEDGEMENTS}

We take this opportunity to express our profound gratitude and deep regards to our guide Prof. Mr. D. D. Pukale for his valuable guidance, monitoring and constant encouragement throughout the course of this work. Also we are obliged to staff members of B.V.C.O.E.W. for the valuable information provided by them in their respective fields. Last but not the least we place a deep sense of gratitude to our family members and our friends who have been constant source of inspiration during the preparation.

\section{REFERENCES}

[1]. Schaefer, G., An introduction to content - based image retrieval, Digital information management (ICDIM), 2013, $8^{\text {th }}$ international conference, ISBN number 978-1-47990613-0, 10-12 September 2013.

[2]. Yogita Mistry, Dr. D.T.Ingole, Survey on Content Based Image Retrieval Systems, International Journal of Innovative Research in Computer and Communication Engineering, ISSN number 2320-9801, Vol. 1, Issue 8, October 2013. [3]. Madhura C., Dheeraj D., Feature Extraction for image retrieval using colour spaces and GLCM, International journal of innovative technology and exploring engineering, ISSN number 2278-3075, Vol. 3, Issue - 2, July 2013.

[4]. Raman Maini, Dr. Himanshu Aggarwal, Study and comparison of various image edge detection techniques, International journal of image processing (IJIP), Vol. (3), and Issue 1.

[5]. G. T. Shrivakshan, Dr. C. Chandrasekar, A comparison of various edge detection techniques used in image processing, International journal of computer science issues (IJCSI), ISSN number 1694-0814, Vol. 9, Issue 5, No 1, September 2012.

[6]. Biman Debbarma and Dibyendu Ghoshal, A modified canny edge detection algorithm with variable sigma, International journal of engineering trends in electrical and electronics (IGETEE), Vol. 1, Issue 2, March 2013.

[7]. S. Sulochana, R. Vidhya, Texture based image retrieval using framelet transform - Gray level co - occurrence matrix, International journal of advanced research in artificial intelligence (IJARAI), Vol. 2, Issue 2, 2013.
[8]. Reena Pagare and Anita Shinde, A Study on Image Annotation Techniques, International journal of computer applications, ISSN number 0975-8887, Vol. 37, No. 6, January 2012.

[9]. Mohd. Danish, Ritika Rawat, Ratika Sharma, A Survey: Content based image retrieval based on colour, texture, shape and Neuro fuzzy, International journal of engineering research and applications, ISSN number 2248-9622, Vol. 3, Issue - 5, Sept - Oct 2013.

[10]. B. Szanto, P. Pozsegovics, Vamossy, Sz. Sergyan, Sketch4Match - Content based image retrieval system using sketches, Applied machine intelligence and informatics (SAMI), 2011, IEEE $9^{\text {th }}$ international symposium, ISBN number 978-1-4244-7429-5, 27-29 January 2011.

[11]. Mr. Vivek Singh Rathore, Mr. Messala Sudhir Kumar, Mr. Ashwini Verma, Colour based image segmentation using $L^{*} A^{*} B^{*}$ colour space based on genetic algorithm, International journal of emerging technology and advanced engineering, ISSN number 2250-2459, Vol. 2, Issue 6, June 2012.

[12]. Neetesh Gupta, Dr. Vijay Anant Athavale, Image retrieval using collaborative approach based on random walk and similarity matching by Hybrid graph representation, International Journal of Emerging Technology and Advanced Engineering, ISSN 2250-2459, Volume 2, Issue 2, February 2012.

[13]. T. Sumathi, C. Lakshmi Devasena and M. Hemalatha, An overview of automated image annotation approaches, International journal of research and reviews in information sciences, Vol. 1, No. 1, March 2011.

\section{BIOGRAPHIES}

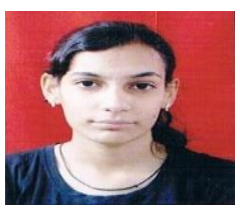

Miss. Nutan D. Pawar., Deaprtment of Computer Engineering, Bharati Vidyapeeth's College Of Engineering for Women, Pune. E - mail ID: pawarnutan74@yahoo.in

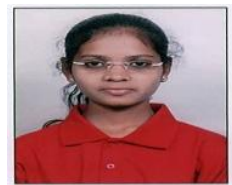

Miss. Varsha H. Sakore. Deaprtment of Computer Engineering, Bharati Vidyapeeth's College Of Engineering for Women, Pune. E - mail ID: sakorevarsha@hotmail.com

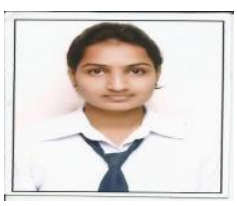

Miss. Priyanka N. Shendage., Deaprtment of Computer Engineering, Bharati Vidyapeeth's College Of Engineering for Women, Pune. Email ID: priyankashendage92@gmail.com

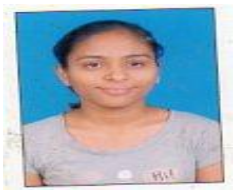

Miss. Nilima K. Shevate, Deaprtment of Computer Engineering, Bharati Vidyapeeth's College Of Engineering for Women, Pune. E - mail ID: nilimashevate@gmail.com 